\title{
CT scan exposure in children with ventriculo-peritoneal shunts: single centre experience and review of the literature
}

\author{
Gareth M. Dobson ${ }^{1}$ - Arthur K. Dalton ${ }^{2}$ • Claire L. Nicholson ${ }^{1}$ • Alistair J. Jenkins ${ }^{1}$ • Patrick B. Mitchell ${ }^{1}$ • \\ Christopher J. A. Cowie ${ }^{1}$
}

Received: 7 May 2019 / Accepted: 7 August 2019 / Published online: 19 August 2019

(C) The Author(s) 2019

\begin{abstract}
Purpose A computed tomography (CT) scan in childhood is associated with a greater incidence of brain cancer. CT scans are used in patients with ventriculo-peritoneal (VP) shunts in whom shunt dysfunction is suspected. We wanted to assess the CT scan exposure in a cohort of children with VP shunts and attempt to quantify their radiation exposure.

Methods A single-centre retrospective analysis was performed recording CT head scans in children younger than 18 years with VP shunts. Hospital coding data was cross-referenced with electronic records and radiology databases both in our neurosurgery unit and in hospitals referring to it.

Results One hundred and fifty-two children with VP shunts were identified. The mean time with shunt in situ was 5.4 years $( \pm$ 4.61). A mean of $3.33 \mathrm{CT}$ scans (range 0-20) were performed on each child, amounting to 0.65 ( \pm 0.87 ) CTs per shunt year. Based on $2 \mathrm{msv}$ of radiation per scan, this equates to an average exposure of $1.31 \mathrm{msv}$ per child per shunt year.

Conclusion Children who have multiple CT head scans for investigation of possible shunt dysfunction are at a greater risk of developing cancer. We discuss the implications of this increased risk and discuss strategies to limit radiation exposure in children with VP shunts.
\end{abstract}

Keywords Hydrocephalus · Computed tomography · Radiation risk

\section{Abbreviations}

CSF Cerebrospinal fluid

CT Computed tomography

Christopher J. A. Cowie

christopher.cowie@newcastle.ac.uk

Gareth M. Dobson

gareth.dobson@nuth.nhs.uk

Arthur K. Dalton

arthurkdalton@yahoo.co.uk

Claire L. Nicholson

claire.nicholson@nuth.nhs.uk

Alistair J. Jenkins

alistair.jenkins@nuth.nhs.uk

Patrick B. Mitchell

patrick.mitchell@newcastle.ac.uk

1 Department of Neurosurgery, Great North Children's Hospital, Queen Victoria Road, Newcastle upon Tyne, UK

2 Atkinson Morley Regional Neurosciences Centre, St George's Hospital, London, UK
MRI Magnetic resonance imaging

VP Ventriculo-peritoneal

\section{Introduction}

Childhood hydrocephalus is a common neurosurgical presentation, affecting 1-3 per 1,000 births and arises from either acquired or congenital abnormalities in cerebrospinal fluid (CSF) dynamics [35]. Management often involves the use of CSF diversion shunts, the most common of which drain from the ventricles or lumbar cistern into the peritoneum but may instead drain from the ventricles to the lung pleura, right atrium or subgaleal space.

Ventriculo-peritoneal (VP) shunt dysfunction has been reported in up to $40-60 \%$ of patients, with the highest incidence of malfunction within the first year after implantation [24, 30]. In a large cohort of children with shunts, just over half experienced at least one episode of shunt malfunction within 12 years of insertion [30]. With VP shunt dysfunction at an estimated annual incidence of $5 \%$, it is a frequent cause of presentation to 
hospital for investigation and management in those with such a device [28].

Hydrocephalus secondary to shunt malfunction in adults typically presents with the following classic triad of symptoms: headache, vomiting and reduced conscious level. However, in children, the clinical picture is less clear-cut, and symptoms may include more notable neurological deficits such as visual and oculomotor disturbance or seizures, or conversely less specific symptoms such as lethargy, general malaise or nausea [14]. This variability in clinical picture on presentation can make it difficult to differentiate shunt dysfunction in children from other common childhood illnesses and infections, particularly in hospitals where neurosurgical expertise is not readily available. Due to the availability and ease of obtaining computerised tomography (CT) scans and the perceived need to rapidly exclude hydrocephalus secondary to shunt dysfunction, head CT scans have become a frequently used baseline investigation in unwell children with VP shunts, as they allow fast assessment of ventricular size and shunt catheter location [26]. While this practice can be entirely appropriate in the unstable or deteriorating child, the practice of rapid scanning may subject some children with shunts to unnecessary radiation. This can be compounded by the fact that head CT scans in this patient group are often requested hand in hand with 'shunt series' $\mathrm{x}$-rays; three or four plain radiographs (of the head, neck, chest and abdomen) focused on the shunt valve and tubing to exclude shunt fracture, disconnection, migration or incorrect placement. [26] While radiographs confer much less radiation than a head CT scan, the cumulative dose is undoubtedly increased when such investigations are requested.

Recent studies have attempted to quantify the risks of future malignancy from CT scans performed in childhood. A large cohort trial following 680,000 children in Australia by Mathews et al. reported a greater incidence of cancer diagnosis in people undergoing CT scans in childhood [20]. They observed a $24 \%$ higher incidence of cancer in children exposed to a single CT scan compared with those who never had a scan, with a subsequent $16 \%$ increase for each additional scan. Interestingly, those children in the cohort who had received a head CT scan were in even greater danger: the risk of developing brain tumours was shown to be $44 \%$ greater after a head CT scan than in children who had never been scanned. Pearce et al. performed a similar cohort study within the UK. The group focused on the estimated risk of developing either leukaemia or brain cancer in children who had undergone CT imaging before the age of 22 years old. They demonstrated that those children undergoing 2-3 head CTs had nearly triple the risk of developing brain tumour and those who had 5-10 head CTs had triple the risk of developing leukaemia. [25]
Further studies aimed at quantifying the risk of performing CT head scans in children have been analysed by Sheppard et al. in a recent systematic review. The review identified that a single CT head scan in childhood carries a 2.4-fold increased risk of developing a brain tumour compared to children who have not been exposed to a CT head scan. Whilst the increased relative risk is significant, the estimated risk of developing radiationinduced cancer corresponds to around one per 1800 paediatric head CTs [31].

In our study, we aimed to examine the numbers of head CT scans performed in children with VP shunts and to assess how frequently they were performed. Using calculations from other peer-reviewed literature, we then sought to estimate the radiation exposure per 'shunt-year' in each child.

\section{Methods}

We performed a single centre, retrospective study of paediatric patients (0-18 years of age), who presented to our unit with suspected shunt dysfunction over a 5-year period. Paediatric patients undergoing ventriculo-peritoneal, ventriculo-atrial or lumbo-peritoneal shunt insertion or revision surgery were identified using clinical coding records. Patient details were crossreferenced with electronic medical records and the hospital radiology database (PACS). In case children with suspected shunt dysfunction had presented to their local emergency departments and had undergone head CT before being referred to our tertiary service, data was also collected from radiology databases in the district hospitals within our catchment area, to ensure we did not underestimate the numbers of CT head scans performed on each child. The following data was recorded: demographics, shunt insertion date, date of shunt revision, removal or death, numbers of head CT scans performed and where available, recorded indication for scan.

Data analysis included calculation of the number of CT scans per patient and the total time with a shunt device in situ over which those scans were performed. The average number of head CT scans per child per year with shunt (shunt-year) was calculated. An estimation of radiation exposure was made, both per shunted child and per shunt-year, based on an average radiation dose of $2 \mathrm{msv}$ per CT head scan [32]. It was not possible to calculate the actual tissue dose, as the quantitative data on radiation exposure for the various scan protocols and scanners in each hospital was outwith the scope of this retrospective analysis. Lastly, we attempted to determine the percentage of CT scans that preceded surgery for either revision or removal of the shunt.

Using figures from a recent population study in which the risk of developing a future radiation-induced malignancy was estimated, we calculated the increased relative risk for our paediatric shunt population [20].

Statistics were calculated using RStudio [29]. 


\section{Results}

A total of 152 children, 71 male and 81 female, underwent CSF shunt insertion during the 5-year study period. A total of 312 shunt surgeries were performed: 115 children received their first shunt, and 197 existing shunt systems were partially or completely revised. Mean age at insertion was 26 months (range 2 days to 15 years). The indication for shunt insertion varied, with tumour, meningitis, congenital hydrocephalus, spina bifida and intraventricular haemorrhage amongst the most common indication for shunt insertion.

A variety of shunts were inserted; however, the most common form of CSF shunt utilised was a VP shunt. Mean shunt lifespan was 5.4 years $( \pm 4.61)$, from shunt insertion to either shunt removal, patient death or their 18th birthday. Sadly, 13 $(8.5 \%)$ patients died prior to either their 18th birthday or study follow-up.

A total of $443 \mathrm{CT}$ head scans were performed at our tertiary referral centre to assess CSF shunt functioning across the duration of the study, with an average of 3 cranial CTs (range 0 $20)$ per child. This corresponds to $0.55( \pm 0.79)$ CT scans per shunt-year.

We obtained data from local units for 79 of the 152 patients (52\%), identifying a further $63 \mathrm{CT}$ head scans or on average a further 0.79 scans per child. Overall, the total number of CT head scans was 506, an average of 3.33 per child. Additional local hospital exposure leads to an increase in the number of CT heads performed per shunt-year in this population group to $0.65( \pm 0.87)$.

We were able to calculate the age at which CT scans were performed for all in-house scans, this ranged from 0 to 18 years, with a mean age of 6 years old. The highest number of CT scans were however seen in those children aged 0-1 year (Fig. 1). A general trend demonstrating a higher number of CT scans performed in patients who had their shunt inserted at a younger age was observed $(p=0.086)$, with those children undergoing insertion of shunt prior to 25 months of age being exposed to the highest number of CT scans (Fig. 2). A significant correlation between numbers of CT scans and number of shunt-years was observed $(p=<0.05)$, with a greater number of CT scans performed in those children who had a shunt in place for a longer period of time (Fig. 3). There appeared to be two populations in this dataset, one in which number of CTs appeared to correlate with shunt-years, and a further population in which no CT scans were performed for the first 5 years after shunting (Fig. 3).

Surgical intervention post-CT scan was recorded in $31 \%$ of cases following CT scan, no intervention was recorded following $48 \%$ of CT scans. The remaining $21 \%$ of scans were performed prior to electronic records, and data regarding intervention was unavailable in relation to these scans. Surgical intervention data was only available for 15 of 63 CT scans performed in referring units, of these $40 \%$ of CT scans led to surgical intervention.

Based on a radiation dose of $2 \mathrm{msv}$ per scan, our children with CSF shunts were exposed to on average $6.66 \mathrm{msv}$ (range $0-40 \mathrm{msv}$ ) of radiation or $1.31 \mathrm{msv}$ per shunt-year. According to the study by Matthews et al., this would correlate to an increased incidence rate ratio (IRR) of 1.61 for developing a future radiation-induced malignancy.

\section{Discussion}

In this retrospective study, we aimed to quantify the number of CT head scans performed in children with suspected CSF shunt malfunction. Our results demonstrate that, on average,
Figure 1 Number of CT head scans performed per child for each year of age throughout the period of the study

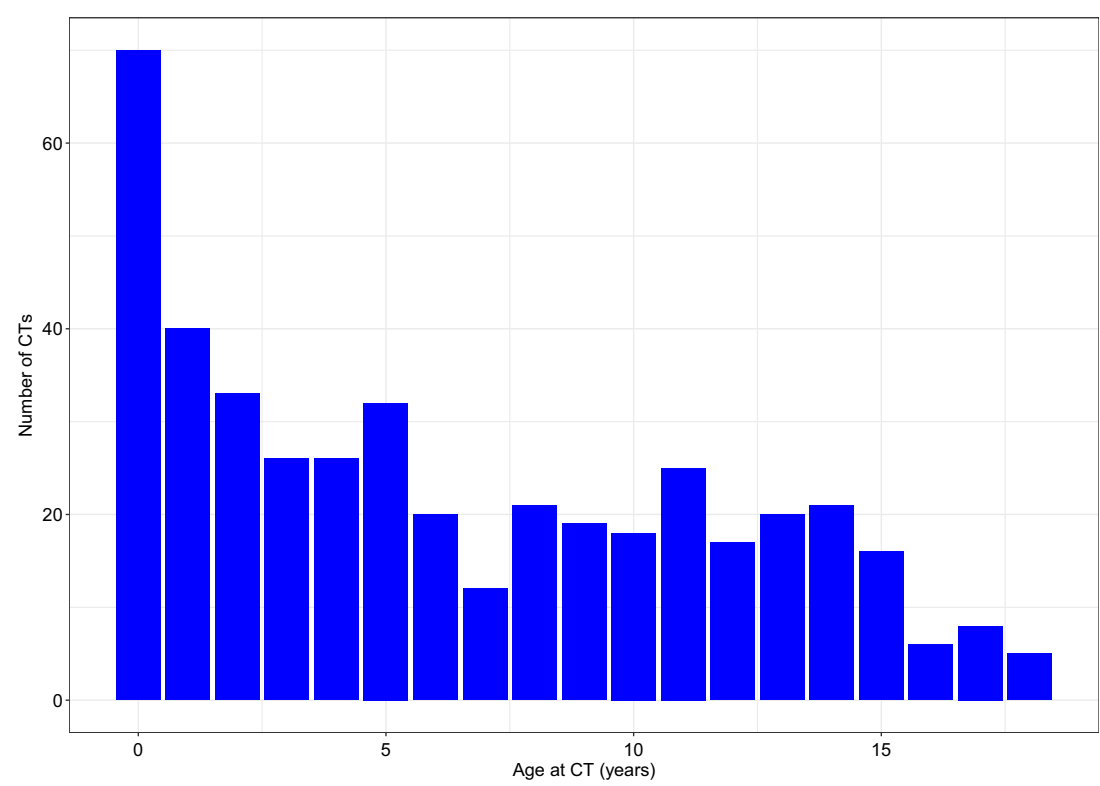


Figure 2 Number of CT scans performed based on age at shunt insertion

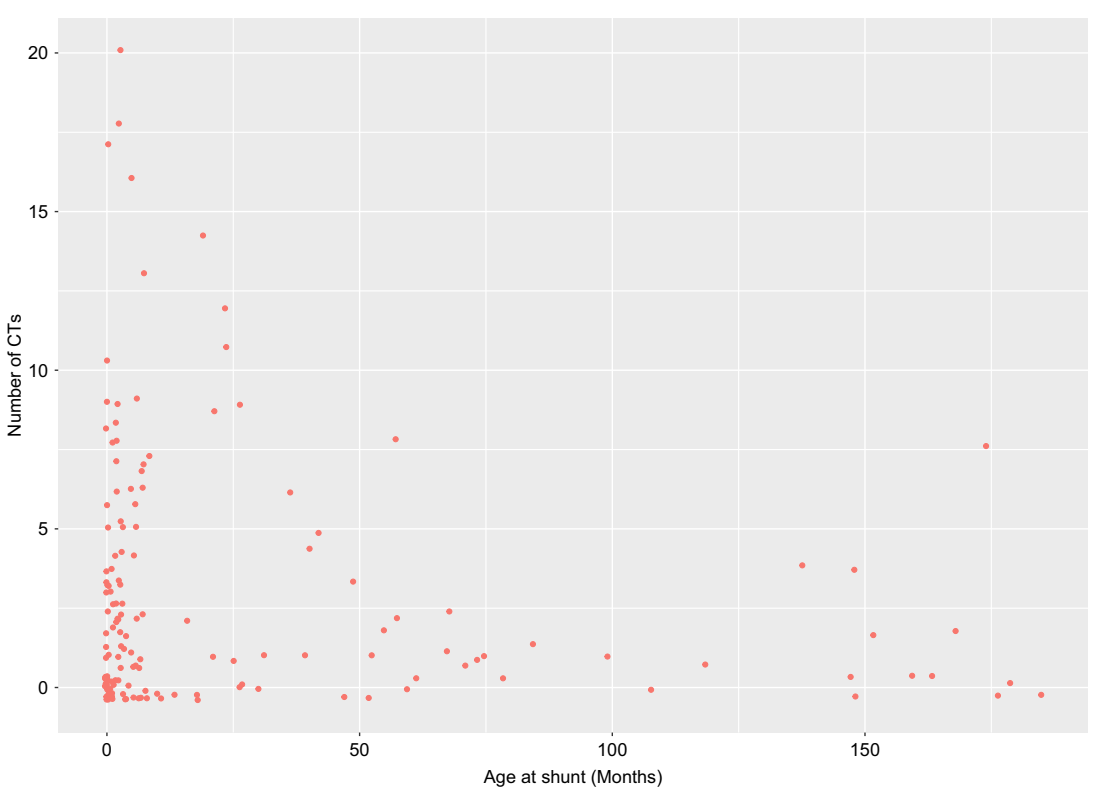

0.65 CT heads were performed per shunt-year, with each child undergoing 3.3 scans on average during the observed period.

CSF shunts, in particular, VP shunts, are frequently used for treatment of childhood hydrocephalus. Despite being the most common treatment for this condition, they are associated with a high rate of malfunction, with one study reporting revision rates of $32.5 \%$ in adults, and significantly higher $(78.2 \%)$ rates of revision observed in children [27]. Stone et al. reported similar revision rates in children with $84.5 \%$ of patients requiring shunt revision and $4.7 \%$ of patients requiring greater than 10 revision procedures, equating to around 2.66 revisions per patient [34].

In general, children with shunt malfunction do present with the expected symptoms of headache, vomiting and drowsiness, but in this young age group, these symptoms can also represent many other childhood illnesses [5]. Cohen et al. failed to demonstrate any correlation between a variety of symptoms, including those mentioned above, and radiographic evidence of shunt malfunction [9]. However, they revealed a positive association between fontanelle swelling and behavioural change and shunt revision, suggesting that clinical presentation alone may provide a valid indication for shunt revision in children with an open fontanelle. In our study, $25.3 \%$ of all CT scans were performed in children less than 2 years of age, suggesting radiation exposure in this sub-population could potentially be reduced through clinical assessment. Another possible adjuvant to help reduce radiation exposure is the use of diagnostic scales. Munoz-Santanach and colleagues recently performed a prospective assessment of children with suspected shunt malfunction presenting to the emergency department and devised a 20-point scale aimed at
Figure 3 Number of CT scans performed based on number of shunt-years

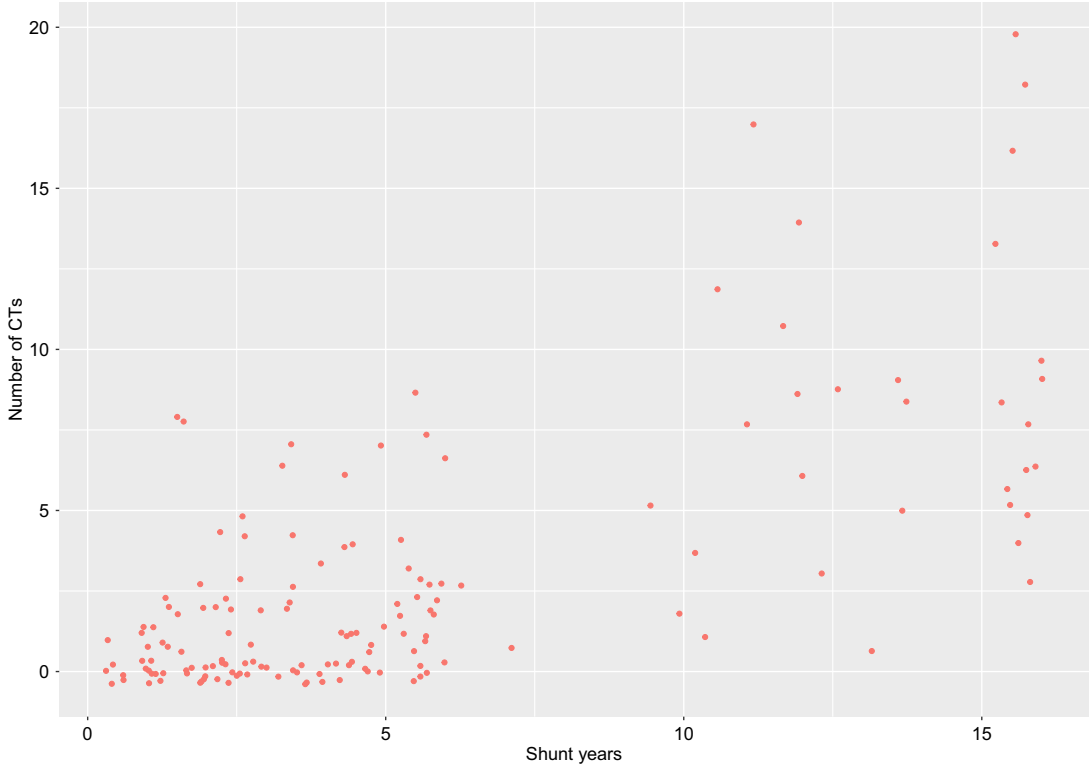


predicting shunt malfunction. Based on various signs and symptoms they had identified, children scoring 7 or more points on the scale were associated with an increased risk of shunt malfunction (PPV 53.4\%, NPV 96.7\%) [22].

CT scans are widely used for both diagnostic and surveillance purposes. In a single centre retrospective review, Lee and colleagues demonstrated high use of CT scans among children with a variety of medical conditions. Over a 5-year period, over $25,000 \mathrm{CTs}$ were performed on a total of 13,800 children, $34.3 \%$ of which were under 1 year at the time of scan. Disease group appeared to be one of the main contributing factors to the number of CT scans performed: children with VP shunts being exposed to a high number of scans [16]. This was reflected in a 10-year retrospective review of emergency department attendance by Florin et al. who identified that 1,319 children with shunts were responsible for 6,636 visits. Of these presentations, $49.4 \%$ resulted in children undergoing CT head scans [11]. There has been an increase in the use of CT imaging over the past 10 20 years, with numbers doubling in children younger than 5 years of age and tripling in children 5 to 14 years of age, between 1996 and 2005 [21]. Our experience within a tertiary referral centre appears to reflect this trend, with a notable, yet anecdotal increase in CT scans being performed by local hospitals in patients with suspected shunt malfunction prior to referral. We demonstrated children with shunts had on average 3.3 CT scans, with a large overall range from 0 to 20 scans per individual child over the period of our study. Higher rates of CT scanning have previously been reported, with one study reporting children with shunts being exposed to 2.6 CT head scans on average per year. [9] Interestingly, White et al. also report high numbers of CT scans in children, with even higher rates in neonates and infants undergoing shunt insertion before the age of 1 year, compared with those whose shunt was inserted between 1 and 6 years of age. [37] This trend is reflected in our own data and is most probably due to a previously identified correlation between younger age at the time of shunt insertion and increased rates of malfunction and infection. [1, 10]

Of the children undergoing 'in house' CT head scans in our study, surgical intervention was observed following $31 \%$ of scans. In a retrospective review of CT scans in children with VP shunts, Antonucci et al., 130 children underwent 1,411 CT head scans, (8.5/pt. median), following which 237 (17\%) underwent surgical intervention within 7 days. [3] Similarly, other studies have also reported slightly lower intervention rates of 13.5 to $30 \%$ of CT scans leading to surgical intervention $[9,19]$.

The use of CT scans in children has consistently raised concern regarding the increased risk of radiation-induced malignancy. Children with VP shunts appear to be exposed to higher levels of radiation compared with children without a shunt. Smyth et al. described two cases of children with VP shunts both of whom had multiple CT scans (14 CTs/23CTs) and subsequently developed malignancies (Hodgkin's lymphoma and gliosarcoma) [33]. However, White et al. performed a retrospective cohort study, including 104 children who underwent CSF shunt placement. In total, 1,584 CT head scans were performed over a 10year period, with no observed malignancies within the cohort [37]. Despite these findings appearing reassuring, there is strong epidemiological evidence linking CT scanning in childhood with an increased risk of malignancy. [8, 31]

Confidently attributing cancer to childhood CT radiation exposure is difficult; however, numerous studies aiming to assess this risk have been published. (Table 1) These include three population studies, reporting patient exposure and outcome data, as well as studies calculating a predicted risk based on the previous population studies or those relating to Japanese Bomb survivors or the BIER reports $[8,13,20,25]$. Pearce et al. were the first group to publish a population study, performing a retrospective cohort study assessing the risk of developing leukaemia or a brain tumour following childhood exposure to CT scanning. Overall, 74/178,604 patients developed leukaemia, estimated relative risk 3.18 (95\% CI, 1.46-6.94) for those exposed to > 30 mGy, and 135/176,587 developed a brain tumour, estimated relative risk 3.32 (95\% CI, 1.84-6.42) for those exposed to > 50 mGy [25]. In their discussion, the authors suggest that children exposed to $>60 \mathrm{mGy}$ radiation may have triple the risk of developing a brain tumour. Mathews et al. then published the largest population study to date, another historical cohort study including over 10 million subjects, of which 680,211 were exposed to CT scan [20]. They observed an incidence rate ratio (IRR) of 1.24 for developing any form of radiation-induced malignancy following a single CT scan, with subsequent 0.16 IRR for each additional CT scan. Sub-analysis of CT brain scans revealed an even higher IRR of 2.44 (2.12-2.81). A further smaller population cohort study demonstrated significantly increased incidence of brain tumours in children exposed to brain CT, Hazard ratio 2.97 (95\% CI, 1.49-5.93). [13]

In a follow-up to the paper from Pearce et al., Berrington de Gonzalez et al. estimated a mean lifetime risk of developing cancer after a head CT scan as between 4 and 9 per 10,000 in children, significantly greater than the risk in adults [6]. A further study solely assessing CT scans in children with shunted hydrocephalus estimated shunt patients undergo 2.1 ( \pm 1.6$)$ head CT scans per year, with one subsequent excess lifetime cancer for every 97 patients [15]. Applying the projected risk figures from the paper by Mathews et al. to data from our study would indicate that children with VP shunts are 1.61 times more likely to develop radiation-induced malignancy [20]. Whilst the overall numbers of radiation-related malignancies will remain relatively low, the additional risk associated with scanning within this population is significant.

Exposure to radiation via CT scans not only increases this risk of malignancy but has also been demonstrated to increase the rate of cataract formation in a dose-dependent fashion. Yuan et al. report an increased incidence in those undergoing more than five CT scans $(2.12 \%)$ compared with those undergoing only one or two scans $(0.79 \%)$ [39]. 


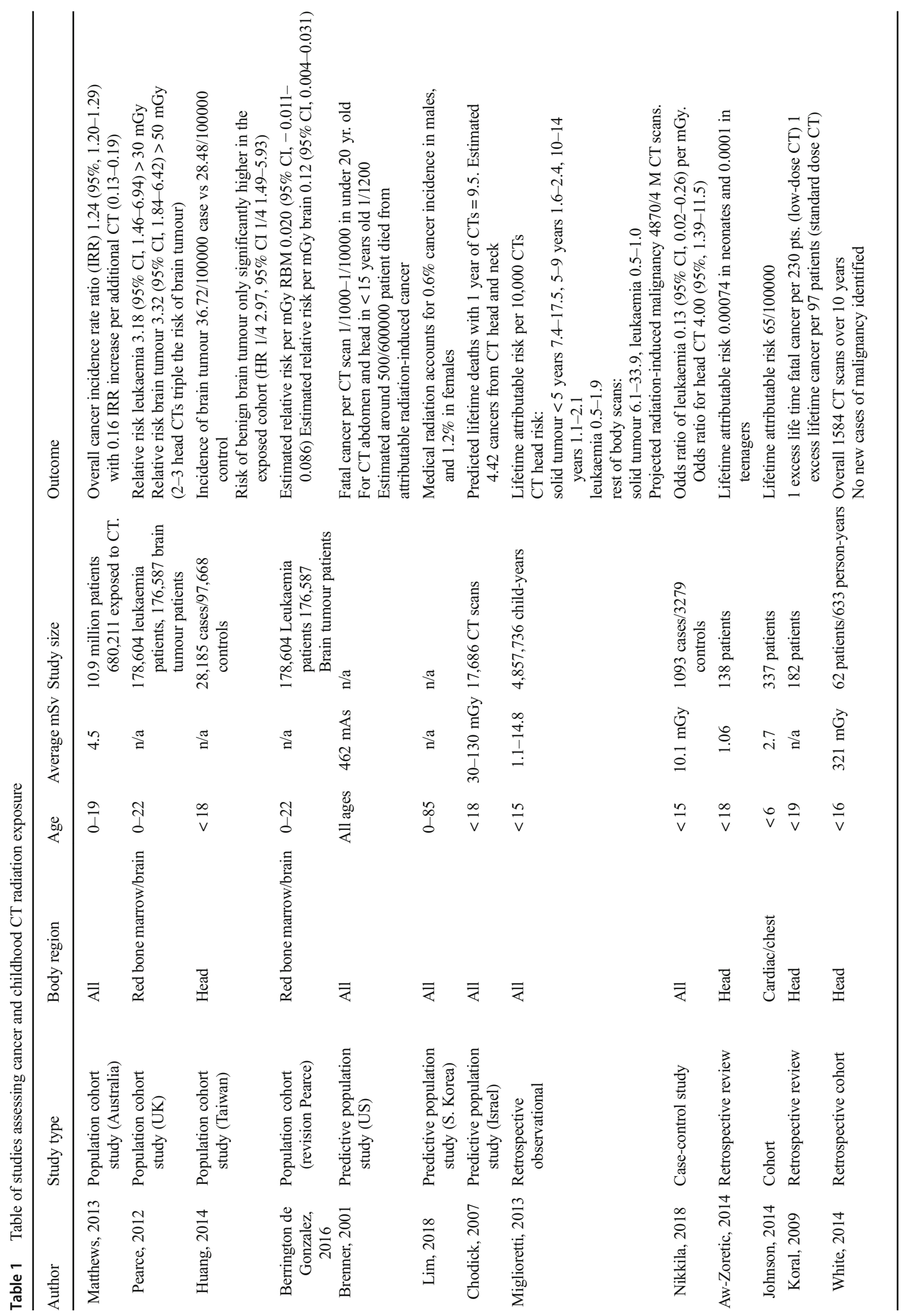


It is not surprising that multiple studies have looked at potential methods for reducing radiation exposure in children. Udayasankar et al. compared the utility of low-dose versus standard-dose CT scans for routine follow-up in CSF shunted patients, reducing radiation exposure by $63 \%$ on average [36]. Despite increased noise, low dose CT scanning yielded diagnostically acceptable imaging. Reduced slice imaging provides a further option, reducing radiation exposure by greater than 90\%. Sensitivity of three-slice CT in identifying the ventricular system and locating CSF shunt tips has been reported as around $92 \%$ and $94 \%$ respectively [2]. Both low-dose scanning and reduced slice scans offer a feasible alternative to complete CT head scans and could be implemented in the diagnostic process for shunt malfunction.

Magnetic resonance imaging (MRI) may offer a further option for investigating suspected shunt malfunction in children. Boyle et al. demonstrated MRI scanning to be of comparable diagnostic accuracy compared with CT scan (81.8\% vs $82.4 \%$ ) in the acute setting of suspected shunt malfunction [7]. MRI image quality has similarly been demonstrated to be excellent or good in $94 \%$ of scans performed for assessment of shunt functioning. In a review of $67 \mathrm{MRI}$ scans performed in children with hydrocephalus, Ashley et al. reported identification of shunt catheter tip in over $75 \%$ of cases, with good to excellent image quality in around $60 \%$ cases [4]. However, the average time of scan was $22 \mathrm{~min}$, over double the average for a CT scan. Young children in particular find long MR acquisition times difficult to tolerate. More recently, faster MRI sequences have been developed to try and overcome this problem [38]. Further limitations to the use of MRI scanning in suspected shunt malfunction would be both the cost, as well as access to an MRI scanner.

Both White et al. and our study demonstrated higher levels of radiation exposure in children under the age of 1 year [37]. Ultrasound examination provides a non-radiating method of assessment of ventricular size in children with suspected shunt malfunction who have a patent fontanelle, and this has been shown to be a reliable method of follow-up in this population by Mandiwanza et al. [17]. Ultrasound has been utilised in other studies to measure optic nerve sheath diameter (ONSD) in paediatric patients with suspected raised ICP [18]. Normal values in the paediatric population have been published, with some studies reporting sensitivities and specificities of $61 \%$ and $22 \%$ respectively, in detecting shunt malfunction in the paediatric population using ONSD measurements [12]. A recent cross-sectional study performed across 33 units reported notable reductions in the use of CT scanning in paediatric population for a variety of medical presentations, including an increase in the use of USS and MRI in patients with ventricular shunts. Whilst this is a positive step, CT scanning remained the most common imaging modality [23].

Overall, high rates of shunt malfunction in children, in conjunction with often non-specific symptoms associated with the condition, undoubtedly contribute to the high levels of CT scans performed within this population group. We observed an average of 0.65 CT scans per shunt-year in our children. Using previously published epidemiological data, we infer that children with shunts have a 1.61 greater increased relative risk of developing radiation-associated malignancy compared with children without shunts. In attempt to reduce this, we would recommend early discussion with a local neurosurgical unit, and admission followed by a period of observation if clinically appropriate. Careful consideration of CT scanning is needed in children who may require imaging; however, an awareness of the availability of reduced slice CT scan, rapid sequence MRI scan, and ultrasound in younger children will undoubtedly help to reduce radiation exposure.

\section{Compliance with ethical standards}

Conflict of interest On behalf of all authors, the corresponding author states that there are no conflicts of interest.

Open Access This article is distributed under the terms of the Creative Commons Attribution 4.0 International License (http:// creativecommons.org/licenses/by/4.0/), which permits unrestricted use, distribution, and reproduction in any medium, provided you give appropriate credit to the original author(s) and the source, provide a link to the Creative Commons license, and indicate if changes were made.

\section{References}

1. Agarwal N, Shukla RM, Agarwal D, Gupta K, Luthra R, Gupta J, Jain S (2017) Pediatric ventriculoperitoneal shunts and their complications: an analysis. J Indian Assoc Pediatr Surg 22:155-157. https://doi.org/10.4103/0971-9261.207624

2. Alhilali LM, Dohatcu AC, Fakhran S (2013) Evaluation of a limited three-slice head CT protocol for monitoring patients with ventriculoperitoneal shunts. Am J Roentgenol 201:400-405. https://doi.org/10.2214/ajr.12.9110

3. Antonucci MC, Zuckerbraun NS, Tyler-Kabara EC, Furtado AD, Murphy ME, Marin JR (2017) The burden of ionizing radiation studies in children with ventricular shunts. J Pediatr 182:210 216.e211. https://doi.org/10.1016/j.jpeds.2016.11.051

4. Ashley WW, McKinstry RC, Leonard JR, Smyth MD, Lee BC, Park TS (2005) Use of rapid-sequence magnetic resonance imaging for evaluation of hydrocephalus in children. J Neurosurg 103:124 130

5. Barnes NP, Jones SJ, Hayward RD, Harkness WJ, Thompson D (2002) Ventriculoperitoneal shunt block: what are the best predictive clinical indicators? Arch Dis Child 87:198-201

6. Berrington de Gonzalez A, Mahesh M, Kim K-P, Bhargavan M, Lewis R, Mettler F, Land C (2009) Projected cancer risks from computed tomographic scans performed in the United States in 2007. Arch Intern Med 169. https://doi.org/10.1001/ archinternmed.2009.440

7. Boyle TP, Paldino MJ, Kimia AA, Fitz BM, Madsen JR, Monuteaux MC, Nigrovic LE (2014) Comparison of rapid cranial 
MRI to CT for ventricular shunt malfunction. Pediatrics 134:E47E54. https://doi.org/10.1542/peds.2013-3739

8. Chen JX, Kachniarz B, Gilani S, Shin JJ (2014) Risk of malignancy associated with head and neck $\mathrm{CT}$ in children: a systematic review. Otolaryngol Head Neck Surg 151:554-566. https://doi.org/10. 1177/0194599814542588

9. Cohen JS, Jamal N, Dawes C, Chamberlain JM, Atabaki SM(2014) Cranial computed tomography utilization for suspected ventriculoperitoneal shunt malfunction in a pediatric emergency department. J Emerg Med 46:449-455. https://doi.org/10.1016/j. jemermed.2013.08.137

10. Di Rocco C, Massimi L, Tamburrini G (2006) Shunts vs endoscopic third ventriculostomy in infants: are there different types and/or rates of complications? A review. Childs Nerv Syst 22:15731589. https://doi.org/10.1007/s00381-006-0194-4

11. Florin TA, Aronson PL, Hall M, Kharbanda AB, Shah SS, Freedman SB, Alpern ER, Mistry RD, Simon HK, Berry J, Coley BD, Neuman MI (2015) Emergency department use of computed tomography for children with ventricular shunts. J Pediatr 167:1382-1388 e1382. https://doi.org/10.1016/j.jpeds. 2015.09.024

12. Hall MK, Spiro DM, Sabbaj A, Moore CL, Hopkins KL, Meckler GD (2013) Bedside optic nerve sheath diameter ultrasound for the evaluation of suspected pediatric ventriculoperitoneal shunt failure in the emergency department. Childs Nerv Syst 29:2275-2280. https://doi.org/10.1007/s00381-013-2172-y

13. Huang WY, Muo CH, Lin CY, Jen YM, Yang MH, Lin JC, Sung FC, Kao CH (2014) Paediatric head CT scan and subsequent risk of malignancy and benign brain tumour: a nation-wide populationbased cohort study. Br J Cancer 110:2354-2360. https://doi.org/ 10.1038/bjc.2014.103

14. Kirkpatrick M, Engleman H, Minns RA (1989) Symptoms and signs of progressive hydrocephalus. Arch Dis Child 64:124-128

15. Koral K, Blackburn T, Bailey AA, Koral KM, Anderson J (2012) Strengthening the argument for rapid brain MR imaging: estimation of reduction in lifetime attributable risk of developing fatal cancer in children with shunted hydrocephalus by instituting a rapid brain MR imaging protocol in lieu of head CT. Am J Neuroradiol 33: 1851-1854. https://doi.org/10.3174/ajnr.A3076

16. Lee E, Goo HW, Lee JY (2015) Age- and gender-specific estimates of cumulative CT dose over 5 years using real radiation dose tracking data in children. Pediatr Radiol 45:1282-1292. https://doi.org/ 10.1007/s00247-015-3331-y

17. Mandiwanza T, Saidlear C, Caird J, Crimmins D (2013) The open Fontanelle: a window to less radiation. Childs Nerv Syst 29:11771181. https://doi.org/10.1007/s00381-013-2073-0

18. Marin JR, Abo AM, Arroyo AC, Doniger SJ, Fischer JW, Rempell R, Gary B, Holmes JF, Kessler DO, Lam SHF, Levine MC, Levy JA, Murray A, Ng L, Noble VE, Ramirez-Schrempp D, Riley DC, Saul T, Shah V, Sivitz AB, Tay ET, Teng D, Chaudoin L, Tsung JW, Vieira RL, Vitberg YM, Lewiss RE (2016) Pediatric emergency medicine point-of-care ultrasound: summary of the evidence. Crit Ultrasound J 8:16-16. https://doi.org/10.1186/s13089-0160049-5

19. Mater A, Shroff M, Al-Farsi S, Drake J, Goldman RD (2008) Test characteristics of neuroimaging in the emergency department evaluation of children for cerebrospinal fluid shunt malfunction. Cjem 10:131-135

20. Mathews JD, Forsythe AV, Brady Z, Butler MW, Goergen SK, Byrnes GB, Giles GG, Wallace AB, Anderson PR, Guiver TA, McGale P, Cain TM, Dowty JG, Bickerstaffe AC, Darby SC
(2013) Cancer risk in 680000 people exposed to computed tomography scans in childhood or adolescence: data linkage study of 11 million Australians. BMJ 346. https://doi.org/10.1136/bmj.f2360

21. Miglioretti DL, Johnson E, Williams A, Greenlee RT, Weinmann S, Solberg LI, Feigelson HS, Roblin D, Flynn MJ, Vanneman N, Smith-Bindman R (2013) The use of computed tomography in pediatrics and the associated radiation exposure and estimated cancer risk. JAMA Pediatr 167:700-707. https://doi.org/10.1001/jamapediatrics.2013.311

22. Munoz-Santanach D, Trenchs Sainz de la Maza V, Candela Canto S, Luaces Cubells C (2016) Clinical scale for the diagnosis of ventriculoperitoneal shunt malfunction in children in Emergency Department. An Pediatr (Barc) 84:311-317. https://doi.org/10. 1016/j.anpedi.2015.06.009

23. Parker MW, Shah SS, Hall M, Fieldston ES, Coley BD, Morse RB (2015) Computed tomography and shifts to alternate imaging modalities in hospitalized children. Pediatrics 136:e573-e581. https:// doi.org/10.1542/peds.2015-0995

24. Peacock WJ, Currer TH (1984) Hydrocephalus in childhood - a study of 440 cases. S Afr Med J 66:323-324

25. Pearce MS, Salotti JA, Little MP, McHugh K, Lee C, Kim KP, Howe NL, Ronckers CM, Rajaraman P, Craft AW, Parker L, Berrington de González A (2012) Radiation exposure from $\mathrm{CT}$ scans in childhood and subsequent risk of leukaemia and brain tumours: a retrospective cohort study. Lancet 380:499-505. https://doi.org/10.1016/S0140-6736(12) 60815-0

26. Pitetti R (2007) Emergency department evaluation of ventricular shunt malfunction: is the shunt series really necessary? Pediatr Emerg Care 23:137-141. https://doi.org/10.1097/PEC. 0b013e3180328c77

27. Reddy GK, Bollam P, Caldito G (2014) Long-term outcomes of ventriculoperitoneal shunt surgery in patients with hydrocephalus. World Neurosurg 81:404-410. https://doi.org/10.1016/j.wneu. 2013.01.096

28. Rekate HL (1991) Shunt revision: complications and their prevention. Pediatr Neurosurg 17:155-162. https://doi.org/10.1159/ 000120587

29. RStudio Team (2015) RStudio: integrated development for R. RStudio, Inc., Boston, MA URL http://www.rstudio.com/

30. Sainte-Rose C, Piatt JH, Renier D, Pierre-Kahn A, Hirsch JF, Hoffman HJ, Humphreys RP, Hendrick EB (1991) Mechanical complications in shunts. Pediatr Neurosurg 17:2-9. https://doi. org/10.1159/000120557

31. Sheppard JP, Nguyen T, Alkhalid Y, Beckett JS, Salamon N, Yang I (2018) Risk of brain tumor induction from pediatric head CT procedures: a systematic literature review. Brain Tumor Res Treat 6:17. https://doi.org/10.14791/btrt.2018.6.e4

32. Smith-Bindman R, Lipson J, Marcus R, Kim K-P, Mahesh M, Gould R, Berrington de González A, Miglioretti DL (2009) Radiation dose associated with common computed tomography examinations and the associated lifetime attributable risk of cancer. Arch Intern Med 169:2078-2086. https://doi.org/10.1001/archinternmed.2009.427

33. Smyth MD, Narayan P, Tubbs RS, Leonard JR, Park TS, Loukas M, Grabb PA (2008) Cumulative diagnostic radiation exposure in children with ventriculoperitoneal shunts: a review. Childs Nerv Syst 24:493-497. https://doi.org/10.1007/s00381-007-0560-x

34. Stone JJ, Walker CT, Jacobson M, Phillips V, Silberstein HJ (2013) Revision rate of pediatric ventriculoperitoneal shunts after 15 years clinical article. J Neurosurg Pediatr 11:15-19. https://doi.org/10. 3171/2012.9.peds 1298 
35. Tully HM, Dobyns WB (2014) Infantile hydrocephalus: a review of epidemiology, classification and causes. Eur J Med Genet 57:359368. https://doi.org/10.1016/j.ejmg.2014.06.002

36. Udayasankar UK, Braithwaite K, Arvaniti M, Tudorascu D, Small WC, Little S, Palasis S (2008) Low-dose nonenhanced head CT protocol for follow-up evaluation of children with ventriculoperitoneal shunt: reduction of radiation and effect on image quality. Am J Neuroradiol 29:802-806. https://doi.org/10.3174/ ajnr.A0923

37. White IK, Shaikh KA, Moore RJ, Bullis CL, Sami MT, Gianaris TJ, Fulkerson DH (2014) Risk of radiation-induced malignancies from CT scanning in children who underwent shunt treatment before 6 years of age: a retrospective cohort study with a minimum 10-year follow-up. J Neurosurg Pediatr 13:514-519. https://doi.org/10. 3171/2014.2.PEDS12508
38. Woodfield J, Kealey S (2015) Magnetic resonance imaging acquisition techniques intended to decrease movement artefact in paediatric brain imaging: a systematic review. Pediatr Radiol 45:12711281. https://doi.org/10.1007/s00247-015-3333-9

39. Yuan M-K, Tsai D-C, Chang S-C, Yuan M-C, Chang S-J, Chen HW, Leu H-B (2013) The risk of cataract associated with repeated head and neck CT studies: a nationwide population-based study. Am J Roentgenol 201:626-630. https://doi.org/10.2214/AJR.12. 9652

Publisher's note Springer Nature remains neutral with regard to jurisdictional claims in published maps and institutional affiliations. 